\title{
BEST PRACTICE
}

\section{Current concepts for the management of systemic lupus erythematosus in adults: a therapeutic challenge}

\author{
Y loannou, D A Isenberg
}

Postgrad Med J 2002;78:599-606

Systemic lupus erythematosus (SLE) is a chronic, autoimmune rheumatic disease with many clinical presentations typically affecting women of childbearing age. The successful therapy of SLE depends upon treating both symptoms and the underlying inflammation. Both non-pharmacological as well as pharmacological therapies are invariably required. Non-pharmacological therapy includes avoiding over-exposure to sunlight with the use of adequate sunscreen protection, avoiding "live" vaccination if on immunosuppressive agents, adherence to a diet low in saturated fat and high in fish oil, stress avoidance, and smoking cessation. Pharmacological measures revolve around four main classes of drugs: non-steroidal anti-inflammatory drugs, antimalarials, corticosteroids, and cytotoxic agents. Cyclophosphamide and azathioprine are the two most commonly used cytotoxic agents and these in combination with corticosteroids need to be employed early if there is major organ involvement to prevent or minimise irreversible damage. The potential side effects of corticosteroids and cytotoxic agents need constant consideration. The rapid developments in biotechnology of recent years may soon lead to new and more specific therapies for patients with SLE.

See end of article for authors' affiliations

Correspondence to Professor David A Isenberg, Centre for Rheumatology, Department of Medicine, Arthur Stanley House, 40-50 Tottenham Street, London WIP 9PG,

UK; d.isenberg@ucl.ac.uk

Submitted

23 January 2002

Accepted 19 June 2002
$\mathrm{T}$ he term "lupus erythemateux" was first coined by Cazenave and Clausit in 1852 and yet 150 years later this condition continues to challenge the physician's capability to deliver safe and effective therapy. Systemic lupus erythematosus (SLE) is a multisystemic, chronic condition affecting individual patients in diverse ways over a varying course of time. Moreover, potential life threatening organ involvement may not cause symptoms until irreversible damage has occurred. Conversely other features such as fatigue, though not life threatening, is for many the single most debilitating feature of their disease. Thus therapy must be tailored to not only suppress disease activity but also to adequately control symptoms. Furthermore there is a need to use the most appropriate regimen of drugs, often long term, to achieve both these goals while acknowledging potential long term side effects of the agents employed. For example some agents commonly used have potential effects on fertility. Given that SLE typically affects young women of childbear- ing potential the therapeutic goals outlined become all the more challenging.

Furthermore the background psychological and social impact on a young person, previously healthy, developing a potentially serious chronic disease needs to be recognised and addressed by the physician and his/her multidisciplinary team.

Much progress in understanding SLE has been made since antinuclear antibodies were first described in $1957 .{ }^{1}$ Though the exact cause of the disease is not known, it is clear that SLE arises from a combination of genetic, environmental, and hormonal factors, with major dysfunction of the immune system. Some autoantibodies play a pathogenic part. Knowledge of the immunopathogenic mechanisms is rapidly evolving, however the ultimate goal of safe, effective targeted therapy remains elusive. Thus the treatment of SLE continues to rely on broad spectrum immunosuppressive agents with all their inherent difficulties.

This article will focus on the pharmacological and other aspects of managing patients with SLE while considering the therapeutic goals and underlying difficulties outlined above. Finally potential new therapies developed to target key components of the immune system will be discussed.

\section{NON-PHARMACOLOGICAL MANAGEMENT}

General measures maybe taken by the patient to help limit the onset and severity of a flare. Probably the most important for many patients, particularly those with skin involvement, is the avoidance of over-exposure to sunlight. Patients must use sunscreens with protection factor 25 or greater if exposed to ultraviolet radiation. Excessive ultraviolet exposure may not only cause a photosensitive rash, but may also be associated with a more general flare of symptoms.

Vaccination for foreign travel is not contraindicated apart from "live" vaccines for patients on more than $10 \mathrm{mg}$ of prednisolone per day or immunosuppressive agents. The precise nature of the immune response to vaccines differs from that in healthy individuals in that, though quantitatively comparable, qualitative differences exist. $^{2}$

The oestrogen contraceptive pill at high dose should be avoided and the lowest oestrogen dose,

Abbreviations: HRT, hormone replacement therapy; NSAIDs, non-steroidal anti-inflammatory drugs; SLE, systemic lupus erythematosus; TPMT, thiopurine methyltransferase 
Table 1 Drug therapy in SLE

\begin{tabular}{|c|c|c|c|c|}
\hline & NSAIDs & Antimalarials & Corticosteroids & Cytotoxic agents \\
\hline Malaise & - & + & + & - \\
\hline Fever & + & - & + & - \\
\hline Serositis & + & - & + & - \\
\hline Arthralgia & + & + & + & - \\
\hline Arthritis & + & + & + & + \\
\hline Myalgia & + & + & + & - \\
\hline Myositis & - & - & + & + \\
\hline Malar/discoid rash & - & + & + & - \\
\hline Pneumonitis & - & - & + & + \\
\hline Carditis & - & - & + & + \\
\hline Vasculitis & - & - & + & + \\
\hline CNS & - & - & $?^{*}$ & $?$ \\
\hline Renal & - & - & + & + \\
\hline Haemolytic anaemia & - & - & + & + \\
\hline Thrombocytopenia & - & - & + & + \\
\hline Raynauds & - & - & $?$ & $?$ \\
\hline Alopecia & - & - & $?$ & $?$ \\
\hline
\end{tabular}

progesterone only pill, or indeed other methods of contraception encouraged. The use of hormone replacement therapy (HRT) remains controversial with some patients anecdotally linking flares to starting HRT. The effects of exogenous oestrogen in SLE is currently being studied in a multicentre, randomised, placebo controlled trial (Safety of Estrogen in SLE: National Assessment, SELINA study) which is approaching completion and should resolve this area of uncertainty.

Other general measures that maybe taken are the avoidance of stress, rest as appropriate and a low saturated fat, high fish oil containing diet. Patients with SLE have a high incidence of premature deaths from accelerated atherosclerotic disease, ${ }^{3}$ and thus adherence to a more general cardioprotective lifestyle such as cessation of smoking should be strongly encouraged.

\section{PHARMACOLOGICAL MANAGEMENT}

The pharmacological management of patients with SLE presently revolves around four main classes of drugs, often in combination. These are:

- Non-steroidal anti-inflammatory drugs (NSAIDs).

- Antimalarials.

- Corticosteroids.

- Cytotoxic drugs.

The broad indication for the use of these drugs are summarised in table 1 and fig 1 and are discussed in greater depth. Table 2 summarises some of the potential side effects and measures that may be taken to limit these. Generally however recommendations regarding when to commence therapy, initial dose and duration of treatment continue to

Table 2 Major side effects of drugs commonly used in SLE

\begin{tabular}{|c|c|c|}
\hline Drug & Side effect & Notes and possible protective strategies \\
\hline NSAIDs & $\begin{array}{l}\text { Gastrointestinal irritation/ } \\
\text { bleeding }\end{array}$ & $\begin{array}{l}\text { Avoid using NSAIDs in patients with history of gastrointestinal bleed } \\
\text { Co-use gastroprotective agents, for example, proton pump inhibitors } \\
\text { Follow recent NICE guidelines regarding COX-2 specific drugs }\end{array}$ \\
\hline Hydroxychloroquine & Retinopathy (rare) & $\begin{array}{l}\text { Baseline assessment } \\
\text { Retinopathy, usually reversible on stopping drug (regular monitoring may be beneficial) }\end{array}$ \\
\hline \multirow[t]{3}{*}{ Corticosteroids } & Osteoporosis & $\begin{array}{l}\text { Bone density scan quantification. Co-prescribe calcium/vitamin D supplements for most } \\
\text { patients on long term steroids. Some may need oral/IV bisphosphonates }\end{array}$ \\
\hline & $\begin{array}{l}\text { Hypertension } \\
\text { Glucose intolerance } \\
\text { Hirsutism }\end{array}$ & $\begin{array}{l}\text { Monitor regularly and treat with standard antihypertensive agents } \\
\text { Checking urine for glucose is usually sufficient for monitoring }\end{array}$ \\
\hline & Susceptibility to infection & $\begin{array}{l}\text { Particularly if also on azathioprine or cyclophosphamide } \\
\text { Low threshold for aggressive antimicrobial therapy }\end{array}$ \\
\hline \multirow[t]{2}{*}{ Azathioprine } & Bone marrow toxicity & $\begin{array}{l}\text { Initially weekly and subsequently } 4-6 \text { weekly full blood count once stable dose } \\
\text { Usually reversible when drug discontinued } \\
\text { Avoid co-prescribing drugs which potentiate effect, for example, allopurinol. Increased } \\
\text { risk of leucopenia with captopril }\end{array}$ \\
\hline & Liver dysfunction & $\begin{array}{l}\text { Monitor liver function tests as per full blood count } \\
\text { Effect usually reversible when drug discontinued }\end{array}$ \\
\hline \multirow[t]{5}{*}{ Cyclophosphamide } & Bone marrow toxicity & $\begin{array}{l}\text { Nadir in white count occurs } 10 \text { days after infusion. This value can be used to deduce } \\
\text { whether next dose can be safely given or needs to be reduced }\end{array}$ \\
\hline & Haemorrhagic cystitis & $\begin{array}{l}\text { Good hydration (IV fluids if giving IV cyclophosphamide) } \\
\text { Mesna is protective: oral or IV }\end{array}$ \\
\hline & Increased risk of malignancy & $\begin{array}{l}\text { Especially bladder carcinoma or lymphoma } \\
\text { Seen less often with IV pulsed treatment presumably as cumulative dose tends to be less } \\
\text { than oral therapy. }\end{array}$ \\
\hline & Infertility/amenorrhoea & Particularly in women over 30 years \\
\hline & Nausea & Powerful antiemetics such as granisetron often necessary \\
\hline
\end{tabular}




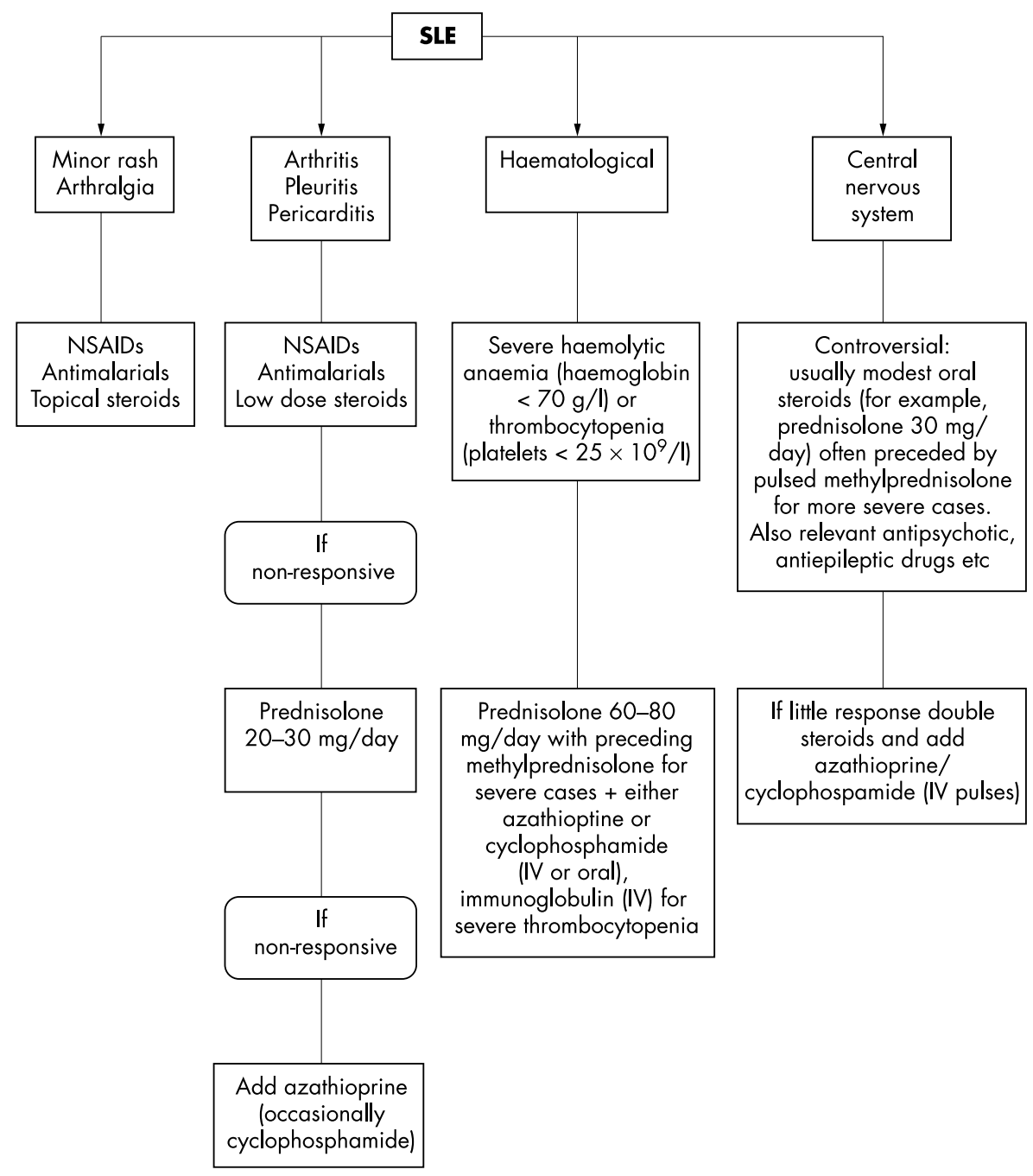

Figure 1 Management of non-renal lupus: practice at our centre. IV, intravenous.

vary widely. This variation in practice has motivated an international effort via the SLICC (Systemic Lupus International Collaborative Clinics group $)^{4}$ to validate existing disease activity measures such as the British BILAG (British Isle Lupus Assessment Group), US SLAM (Systemic Lupus Activity Measure) and Canadian SLEDAI (SLE Disease Activity Index) in addition to formulating a validated damage index. ${ }^{5}$ By more accurately quantifying disease activity and disease damage intervention with appropriate therapy via standardised treatment protocols can lead to a greater quality of care and a greater uniformity in practice between centres. The SLICC group is currently aiming to define a drug responder index for patients with SLE.

\section{Non-steroidal anti-inflammatory drugs and antimalarials}

Patients with mild lupus can generally be maintained on a combination of NSAIDs and antimalarials. NSAIDs significantly increase the risk of developing peptic ulceration. Given that patients with lupus often take NSAIDs regularly, long term appropriate precautions such as co-prescribing gastroprotective agents for example should be considered. Particular caution with regards to the nephrotoxic side effects of NSAIDs is advised in patients with lupus nephritis as this group of patients often have abnormal renal function.

The antimalarial drug of choice is hydroxychloroquine (Plaquenil). This is most commonly employed to treat patients whose over-riding symptoms are those of fatigue, arthralgia/ arthritis, and rash without major organ involvement. A small randomised trial has shown that patients who discontinued hydroxychloroquine were more likely to develop flares often characterised by fatigue, arthralgia, and/or rash. ${ }^{6}$ The starting dose is $400 \mathrm{mg}$ daily, is generally tolerated well and patients should be advised that onset of action may take as long as six to eight weeks. A rare but potentially serious side effect is retinal toxicity with an approximate prevalence of $0.5 \%{ }^{7}$ Most centres thus refer patients to ophthalmologists for baseline screening and have subsequent assessments every 12 months, though a review has proposed that there is no conclusive evidence supporting such frequent assessments. ${ }^{8}$ Hydroxychloroquine has also been shown to help lower total triglyceride levels, very low density lipoprotein-cholesterol, and apolipoprotein CIII levels. ${ }^{9}$

\section{Treatment with corticosteroids}

Relatively low dose corticosteroids are used when NSAIDs and antimalarials have failed to control symptoms of arthralgia/ arthritis or rash sufficiently. When there is major organ involvement with potential life threatening sequelae corticosteroids are used at higher doses (table 1 and fig 1). Examples are autoimmune haemolytic anaemia, lupus nephritis (fig 2), severe pericarditis, and neuropsychiatric involvement.

The route of administration and initial doses varies depending on the type and severity of presentation and different centres probably differ in their precise protocol. Generally corticosteroids are taken orally with the dose varying according to severity and type of organ involvement. Intramuscular methylprednisolone (Dep-Medrone) or hydrocortisone (Deltastab) maybe useful in terminating a mild flare characterised 


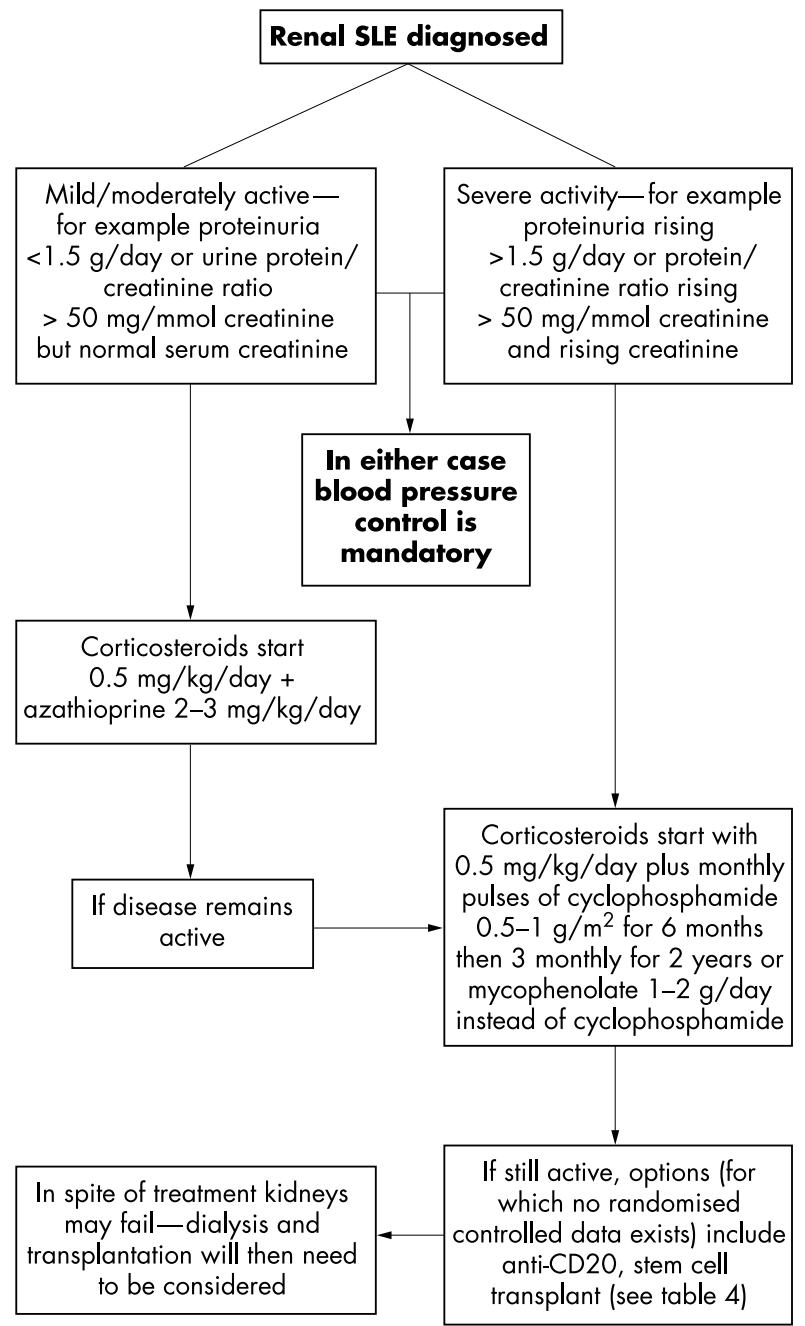

Figure 2 Management of renal lupus based on our own current practice.

by fatigue and/or arthralgia, which may be precipitated by a preceding viral infection for example. Intravenous use of pulsed methylprednisolone has been widely used for the past 20 years. Our practice is to give $750 \mathrm{mg}$ to $1 \mathrm{~g}$ on three successive days for patients with severe disease responding poorly to oral prednisolone. The infusion is given slowly over 3-4 hours to minimise possible reactions such as arthritis, flushing, headache, or tachycardia.

Many patients with SLE need aggressive, often long term therapy with corticosteroids to preserve major organ function. Hence many are exposed to the potentially hazardous side effects of corticosteroids and are thus no panacea (table 2). The long list of side effects includes major risk of infection, hyperlipidaemia, hypertension, osteoporosis, diabetes, insomnia, and alopecia. All of these side effects may have significant and specific implications in a patient with active lupus as follows: infection is a leading cause of mortality in patients with lupus, ${ }^{10}$ patients with active SLE have a high incidence of accelerated atherosclerotic disease, it is vital that hypertension is aggressively controlled in patients with lupus nephritis, patients with premature ovarian failure secondary to cytotoxic therapy are at significant risk of severe osteoporosis. Thus the treating physician must be alert to all these potential hazards, which may be exacerbated by indiscriminate use of high dose corticosteroids.

The issue of osteoporosis needs to be addressed in some detail as cross sectional studies have identified low bone mineral density in SLE patients when compared with age matched controls. ${ }^{11}$ Patients with SLE are at significant risk of developing osteoporosis through several factors: the inflammatory disease itself, disease related co-morbidity, and its treatment. Hence all patients should be assessed for additional risk factors for osteoporosis and general lifestyle measures adopted: these include smoking cessation, moderate consumption of alcohol, adequate intake of dietary calcium and vitamin $\mathrm{D}$, and regular weight bearing exercise. ${ }^{11}$ The relationship between corticosteroids and osteoporosis has been specifically examined in SLE in several studies. Risk of fracture is related to length of treatment ${ }^{12}$ and cumulative exposure. ${ }^{13}$ Hence the practice at our centre for patients taking prednisolone $7.5 \mathrm{mg}$ daily or more is to start calcium and vitamin D supplements, advocate general "bone protective" lifestyle measures, and perform a bone mineral density scan every two years approximately. Several randomised controlled studies have confirmed the efficacy of bisphosphonates in treating corticosteroid induced bone loss and reducing risk of vertebral fractures. ${ }^{14-16}$ Thus patients with a bone mineral density T score of below -2.5 should be prescribed a bisphosphonate unless contraindicated for greater bone protection. We do not routinely prescribe HRT for patients with SLE and osteoporosis due to the uncertain risk of flare and the presence of alternative, effective antiresorptive agents.

\section{Cytotoxic drugs}

The onset of active lupus with major organ involvement is often rapid and requires prompt, aggressive therapy with cytotoxic agents, preferably by specialists to prevent potential disastrous sequelae. Moreover, the frequency with which this occurs in a given population base such as an average catchment area of 250000 though not rare, is uncommon. Thus the nature of this condition is such that controlled trials are challenging to design and require an international collaborative effort to generate evidence guiding best practice. Placebo controlled trials, though deemed the "gold standard" clearly have no place in this setting. Thus up until the past decade or so many rheumatologists have largely relied on their own individual experience combined with data from small open labelled studies to guide therapy. This is now rapidly changing with greater national and international collaboration between centres.

The mainstay of treating active lupus with major organ involvement is broad spectrum immunosuppression with the ultimate aim of preventing irreversible organ damage. The drugs most frequently employed are azathioprine or cyclophosphamide together with corticosteroids, often in high doses. The kidney is the major organ most frequently threatened by active lupus. The group from the National Institute of Health at Bethesda have argued strongly that intravenous pulses of cyclophosphamide, monthly for six months and subsequently every three months for two years, is the treatment of choice in patients with severe renal involvement ${ }^{17}$ when compared with pulsed methylprednisolone alone. Some evidence supports the combination of pulsed methylprednisolone and cyclophosphamide as being superior to cyclophosphamide alone. ${ }^{18} 19$ The indiscriminate use of cyclophosphamide, however, is limited by its side effect profile (profound nausea, alopecia, infertility especially in patients over the age of 30, bone marrow suppression) and consequently has made others more wary about its routine use. In common with many European groups, we prefer to use oral prednisolone with azathioprine in mild to moderately active renal lupus reserving pulsed cyclophosphamide for severe renal involvement (fig 2). Moreover specific changes on a renal biopsy in histology/immunofluorescence as well as confirming the diagnosis can be used to score a validated activity and chronicity index. Thus a renal biopsy may guide the treating physician in stratifying the level of therapy required. However, before a renal biopsy may be safely 
performed adequate control of blood pressure is mandatory. Such control is a key element in managing lupus nephritis effectively, whatever immunosuppressive regime is chosen. A combination of antihypertensive agents is often required to prevent irreversible loss of renal function.

The early recognition of lupus nephritis combined with appropriate, aggressive immunosuppressive therapy and attention to blood pressure control has meant that very few patients progress to end stage renal failure compared with 20-30 years ago. However there are occasional patients in whom, despite the above measures and good compliance, fail to gain adequate long term control and eventually require renal replacement therapy. Though it is relatively uncommon for a lupus patient with a transplanted kidney to develop lupus nephritis in the transplanted kidney, ${ }^{20}$ as reviewed by Stone et al lupus patients having received a renal transplant do not fare as well as those whose end stage renal disease has other causes. ${ }^{21}$

Azathioprine may also be used as a steroid sparing agent in patients whom reducing the dose of prednisolone, albeit slowly, results in a flare. For example, a patient who experiences pleuritic pain at a dose of prednisolone below $10 \mathrm{mg}$ daily may remain symptom free on $5 \mathrm{mg}$ and azathioprine $100 \mathrm{mg}$ daily. The maximum dose of azathioprine we tend to escalate to is 2-3 $\mathrm{mg} / \mathrm{kg} / \mathrm{day}$, though sufficient control may be gained with lower doses. There is some debate as to whether patients on azathioprine should be genotyped for thiopurine methyltransferase (TPMT). Azathioprine is metabolised to its active metabolite 6-mercaptopurine. This in turn undergoes intracellular conversion to thiogunaine nucleotides that incorporate into DNA, producing an antiproliferative effect. Alternatively mercaptopurine is methylated to inactive metabolites by TPMT. TMPT exhibits genetic polymorphism with about $10 \%$ having intermediate activity and about one in 300 having TPMT deficiency. ${ }^{22}$ These patients are in danger of rapidly accumulating high levels of antiproliferative thioguanine nucleotides in haemopoetic tissues at usually therapeutic doses of azathioprine, hence rendering them at risk of severe marrow suppression. This has led some to advocate routine TPMT genotyping on patients starting azathioprine. ${ }^{23}$ However a study in SLE patients on azathioprine showed that drug related neutropenias occur despite normal TPMT activity ${ }^{24}$ Furthermore TPMT activity cannot predict liver toxicity. Our practice is in line with the British Society of Rheumatology guidelines: (in adults) azathioprine is started at $50 \mathrm{mg}$ a day and increased by $25 \mathrm{mg}$ per week to an eventual maintenance of $2-3 \mathrm{mg} / \mathrm{kg} /$ day. Weekly monitoring blood tests are performed for the first six weeks or until the dose is stable. Monitoring bloods are then done monthly thereafter. Whilst TPMT genotyping may augment azathioprine monitoring, we feel that the above monitoring measures are probably sufficient and do not feel that TPMT typing should replace regular blood monitoring.

Surprisingly, though methotrexate is the first line therapy for rheumatoid arthritis, its potential as a steroid sparing agent in SLE has been infrequently studied. A few studies to date have shown that methotrexate may have a place in the treatment of patients in whom cutaneous and articular manifestations have proved refractory to hydroxychloroquine ${ }^{25}$ and low dose prednisolone. ${ }^{26}$

Mycophenolate mofetil is a relatively new immunosuppressive agent now rapidly establishing a role in treating severe lupus nephritis refractory to other cytotoxic agents. Mycophenolate in combination with prednisolone has been compared to cyclophosphamide/prednisolone combination in the treatment of renal lupus. ${ }^{27}$ Both regimens were equally effective with fewer side effects in the mycophenolate group. Thus the use of this agent is likely to increase in the future and based on current evidence should certainly be considered if cyclophosphamide has failed.

\section{OTHER "STANDARD" TREATMENTS}

\section{Plasma exchange}

The concept of removing presumably pathogenic circulating immune complexes seems at first glance to offer a therapeutic advantage. Thus in the late 1970s and early 1980s there was a great vogue for using plasma exchange. After a short time, however, many disadvantages became apparent. As reviewed elsewhere limitations in its use include a "rebound" phenomenon within a few days/weeks, technical difficulties of requiring central venous access with associated complications, and patient discomfort. ${ }^{28}$ This is also an expensive form of therapy. Even in combination with pulsed cyclophosphamide no additional benefit over pulsed cyclophosphamide alone is seen. ${ }^{29}$

\section{Dietary therapy}

A frequently asked question in clinic is whether dietary modification may help. In the laboratory, altering the course of the disease may be achieved in a lupus mouse model (NZB/W and MRL-lpr/lpr mice). Calorific and in particular fat restriction is beneficial as is total zinc reduction. Moreover fish oil supplementation together with a low saturated fat diet has been shown to be beneficial. This has also been seen in humans where a small study showed the beneficial effects of fish oil supplementation over a six month period. ${ }^{30}$ Calorie restriction, however, has no place in the patient with severely active lupus with major organ damage as these patients tend to be acutely unwell and hence in a hypercatabolic state.

\section{Intravenous high dose gammaglobulins}

Intravenous immunoglobulin has an established role in lupus patients with severe thrombocytopenia or immune neutropenia. When this approach is unsuccessful in treating thrombocytopenia, splenectomy is beneficial in four or five out of six cases provided the problem has not been left to become chronic. ${ }^{31}$ Its role in treating non-haematological manifestations of lupus is less clear. A small open labelled study has shown comparable benefit to cyclophosphamide in treating lupus nephritis, ${ }^{32}$ though this needs to be confirmed in larger randomised controlled studies.

\section{Cyclosporin/Neoral therapy}

Following earlier discouraging results with cyclosporin at high doses, ${ }^{33}$ some recent evidence has demonstrated that cyclosporin in low doses of $2.5-5 \mathrm{mg} / \mathrm{kg} /$ day may provide reasonable disease control and offer the opportunity for reduction in steroid over long term follow up. ${ }^{34}$ However there is an impressive side effect profile, notably hypertrichosis which develops in the majority of patients, and given cyclosporin's nephrotoxicity it is best avoid in patients with significant renal impairment.

\section{MANAGEMENT IN SPECIAL SITUATIONS Pregnancy}

Given that most patients with SLE are women of childbearing potential the question of managing pregnancy is a significant issue for many. Approximately $25 \%$ of patients do not go to full term, well in excess of $10 \%$ expected in healthy women. Those patients with antiphospholipid syndrome are especially likely to have recurrent miscarriages. Those with antibodies to Ro and La have a small chance $(1: 20)$ of having a child with neonatal lupus syndrome. In contrast to patients with rheumatoid arthritis those with lupus do not invariably become less active during pregnancy, though whether there is a greater incidence of flares is controversial. Thus careful monitoring is mandatory and on occasion pre-eclamptic toxaemia may be difficult to distinguish from a flare. We strongly recommend co-managing pregnant SLE patients with an interested obstetrician.

Clearly dilemmas arise in pregnant lupus patients regarding appropriate treatment to control active disease. NSAIDS, hydroxychloroquine, high dose corticosteroids, and cytotoxic 
Table 3 Effects of medication in pregnancy

\begin{tabular}{|c|c|c|}
\hline Drug & Known effects on fetus & Current advice \\
\hline High dose aspirin and NSAIDs & $\begin{array}{l}\text { Possible adverse effects on uterine } \\
\text { contraction and platelet function. } \\
\text { Patent ductus arteriosus }\end{array}$ & $\begin{array}{l}\text { Avoid if possible-particularly final } \\
\text { few weeks of pregnancy }\end{array}$ \\
\hline Hydroxychloroquine & $\begin{array}{l}\text { Withdrawal during pregnancy } \\
\text { associated with flares }\end{array}$ & $\begin{array}{l}\text { Chloroquine but not } \\
\text { hydroxychloroquine associated with } \\
\text { congenital abnormalities and so best } \\
\text { avoided }\end{array}$ \\
\hline Corticosteroids & $\begin{array}{l}\text { High doses associated with } \\
\text { premature rupture of membranes, } \\
\text { intra uterine growth retardation and } \\
\text { maternal complications such as } \\
\text { gestational diabetes, hypertension } \\
\text { and avascular necrosis. No reports of } \\
\text { teratogenicity }\end{array}$ & $\begin{array}{l}\text { If possible avoid fluorinated } \\
\text { corticosteroids such as } \\
\text { dexamethasone and betamethasone } \\
\text { which easily cross the placenta }\end{array}$ \\
\hline Azathioprine & $\begin{array}{l}\text { Teratogenicity reported in animals. } \\
\text { Sporadic cases such as preaxial } \\
\text { polydactyl reported in humans. Most } \\
\text { studies show that generally well } \\
\text { tolerated }\end{array}$ & $\begin{array}{l}\text { Best avoided but may be considered } \\
\text { if immunosuppression deemed } \\
\text { necessary }\end{array}$ \\
\hline Cyclophosphamide & $\begin{array}{l}\text { Teratogenicity well established in } \\
\text { humans }\end{array}$ & $\begin{array}{l}\text { Avoid during pregnancy. Effective } \\
\text { contraception required during and for } \\
\text { at least } 3 \text { months after discontinuation } \\
\text { of treatment (includes males) }\end{array}$ \\
\hline Mycophenolate mofetil & Toxicity seen in animal studies & $\begin{array}{l}\text { Avoid during pregnancy. Effective } \\
\text { contraception required during and for } \\
6 \text { weeks after discontinuation of } \\
\text { treatment }\end{array}$ \\
\hline Cyclosporin A & $\begin{array}{l}\text { Not an animal teratogen. Studies } \\
\text { have confirmed relative safety in } \\
\text { pregnancy. Long term effects } \\
\text { uncertain }\end{array}$ & $\begin{array}{l}\text { May be considered if } \\
\text { immunosuppression deemed } \\
\text { necessary }\end{array}$ \\
\hline
\end{tabular}

agents are all best avoided during pregnancy according to the British National Formulary. However one must weigh potential benefits against risk and discuss possible options with the patient. Some drugs carry a low risk of problems while others like cyclophosphamide should be avoided altogether. The challenging area of pregnancy in lupus is reviewed by Mok and Wong, ${ }^{35}$ and current prescribing advice regarding commonly used drugs in lupus is summarised in table 3.

\section{Secondary antiphospholipid syndrome}

Approximately $10 \%$ of SLE patients also have antiphospholipid syndrome (though around 25\%-35\% have antiphospholipid antibodies). In some patients antiphospholipid syndrome may be the predominant clinical phenotype and we have, rarely, seen patients deemed to have primary antiphospholipid syndrome develop SLE over time. In the main, however, our experience is that SLE is diagnosed first. In patients with this type of overlap problems may arise. For example, if a patient develops hypertension with mild proteinuria a lower threshold for biopsy is required to differentiate multiple small thrombi from "lupus" glomerulonephritis. The former requiring anticoagulation and the latter immunosuppression. The difficulties are compounded by the fact that patients with antiphospholipid syndrome are often thrombocytopenic. In some both immunosuppressives and long term anticoagulation are required.

\section{Drug induced lupus}

There is an ever growing list of drugs reported to induce lupus or "lupus-like" disease. Thus far in excess of 50 drugs have been reported, some well known and associated with a relatively high risk such as procainamide, hydralazine or phenytoin, and others not so well known and associated with relatively low risk, such as atenolol, enalapril, or statins for example. ${ }^{36}$ Drug induced lupus is associated with the presence of antihistone antibodies, reported in some studies to be present in up to $100 \%$ of patients. Patients may be taking the offending drug for months or years before developing drug induced lupus and this should not be confused with the short term toxic effects that often are suffered by patients who are treated with pharmaceuticals.

Treating physicians should always consider this potential diagnosis, particularly in patients with atypical presenting features and taking other medications. If suspected, we would advocate a relatively low threshold for requesting antihistone antibodies and performing a literature search. Serum may need to be sent to a dedicated laboratory to perform antihistone antibody quantification and this often needs to be discussed with one's own immunology department.

The mainstay of treatment is discontinuation of the offending drug once the diagnosis is established. Improvement and permanent resolution of symptoms often occurs within days or weeks after discontinuation. ${ }^{36}$ Serological abnormalities often take months to resolve. Patients may need to be on corticosteroid therapy if there is major organ involvement. However the prolonged use of corticosteroids or cytotoxic agents is not indicated in drug induced lupus in view of its self limiting nature after discontinuing the offending drug.

Finally it is worth mentioning the striking association between the use of tumour necrosis factor-alpha blockade therapy and the emergence of dsDNA autoantibodies. This therapy is now used widely for the treatment of severe, refractory rheumatoid arthritis. Studies have shown that around $10 \%$ of patients develop anti-dsDNA antibodies, ${ }^{37}$ although only a very small minority of these patients go on to develop clinical evidence of SLE. Hence though the development of these antibodies in patients on tumour necrosis factor-alpha 
Table 4 Potential new targeted therapies for SLE

\begin{tabular}{|c|c|c|c|}
\hline Drug & Target & Rationale & Trial data available \\
\hline LP 394 & $B$ cells & $\begin{array}{l}\text { Inhibit production of anti-dsDNA antibodies. } \\
\text { Cross links anti-dsDNA antibodies leading to } \\
\text { anergy and deletion of B cells rather than } \\
\text { activation, as drug does not carry T cell epitope } \\
\text { and cannot recruit T cell help }\end{array}$ & $\begin{array}{l}\text { To date no evidence of reduced rate of renal flare. } \\
\text { Phase II clinical studies to identify precise potential } \\
\text { role continue }\end{array}$ \\
\hline Anti-CD20 antibody & B cells & $\begin{array}{l}\text { Inhibits production of anti-dsDNA antibodies. } \\
\mathrm{CD} 20 \text { expressed on all activated B-cells and when } \\
\text { recognised by anti-CD20 antibody causes lysis of } \\
\text { these cells. } \\
\text { Currently licensed for non-Hodgkins lymphoma }\end{array}$ & $\begin{array}{l}\text { Recent small study has demonstrated saftey and } \\
\text { some efficacr at higher doses. Currently underway } \\
\text { at our centre is an open labelled study. Thus far } 7 \\
\text { patients treated with encouraging results. Data } \\
\text { from larger controlled studies required }\end{array}$ \\
\hline Anti-IL-10 antibodies & IL-10 & IL-10 consistently raised in SLE & $\begin{array}{l}\text { Small study of } 6 \text { patients treated showing } \\
\text { improvement in skin and joint manifestations }\end{array}$ \\
\hline Anti-CD40 ligand (L) & $\mathrm{CD} 40 \mathrm{~L}$ & $\begin{array}{l}\text { C4OL expressed on T cells provides an important } \\
\text { co-stimulus to T cell activation. This interaction also } \\
\text { has significant effects on B cells, for example, } \\
\text { augmenting B cell responses to cytokines and } \\
\text { causing antibody isotype switching }\end{array}$ & $\begin{array}{l}\text { One study found this to be tolerated well. } \\
\text { However a recent study was discontinued due to } \\
\text { concerns regarding major vascular events, namely } \\
\text { myocardial infarction and stroke }\end{array}$ \\
\hline Leflunomide & $T$ and $B$ cells & $\begin{array}{l}\text { Inhibits pyrimidine synthesis thus blocking RNA } \\
\text { and DNA synthesis in T and B cells and hence } \\
\text { inhibits proliferation of these cells. } \\
\text { Currently licensed for rheumatoid arthritis }\end{array}$ & $\begin{array}{l}\text { First short term studies have shown it to be safe } \\
\text { and reasonably effective }\end{array}$ \\
\hline
\end{tabular}

blockade is not an indication in itself to discontinue therapy, patients must be kept under careful review.

\section{POTENTIAL NOVEL THERAPIES}

Rapid developments in biotechnology over the past decade has offered the opportunity to develop a greater understanding of the immunopathogenic dysregulation characterising lupus and develop targeted therapy to interfere with this dysregulation at various levels. Reagents near or at clinical trial phase include biologics developed to modulate or inhibit T cell activation, $\mathrm{T}$ and $\mathrm{B}$ cell collaboration, anti-dsDNA antibody production, deposition of anti-dsDNA antibody complexes, complement activation/deposition, and cytokine activation. In addition autologous stem cell transplantation has been attempted for very severe refractory lupus with remission induced in the majority of the small number of patients recruited..$^{38}$ Some of the new therapies currently under study are summarised in table 4 .

\section{CONCLUSION}

The prognosis of SLE has improved considerably over the past 30-40 years with appropriate use of corticosteroids and cytotoxic agents. Nevertheless the treatment of lupus continues to present considerable challenges. Many have mild disease, while others may present or rapidly develop aggressive disease with irreversible organ damage requiring prompt use of broad spectrum immunosuppressive therapy with their inherent difficulties. Indeed weighing the potential benefits of treating active disease against the risks of therapy induced side effects often represents the greatest dilemma for many and every intervention requires that the patient be fully informed of potential risks. The rapid developments in biotechnology have seen the production of specific targeted therapy, many of which are currently in clinical trial phase. The role of accelerated atherosclerosis as a cause of death in patients with SLE is increasingly recognised and presents further challenges for the future.

\section{Authors' affiliations}

Y loannou, D A Isenberg, Centre for Rheumatology, Department of Medicine, University College London, London, UK

\section{REFERENCES}

1 Holborrow EJ, Weir DM. A serum factor in lupus erythematosus with affinity for tissue nuclei. BM 1957;ii:732-6.

2 loannou $Y$, Isenberg DA. Immunisation of patients with systemic lupus erythematosus: the current state of play. Lupus 1999;8:497-501.

3 Salmon JE, Roman M. Accelerated atherosclerosis in systemic lupus erythematosus: implications for patient management. Curr Opin Rheumatol 2001;13:341-4.

4 Isenberg DA, Gladman D. The Systemic Lupus International Collaborating Clinics Group-origins and outcomes. Lupus 2001;10:375-7.

5 Gladman DD, Urowitz MB. The SLICC/ACR damage index: progress report and experience in the field. Lupus 1999;8:632-7.

6 The Canadian Hydroxychloroquine Study Group. A randomized study of the effect of withdrawing hydroxychloroquine sulfate in systemic lupus erythematosus. N Engl J Med 1991;324:150-4.

7 Levy GD, Munz SJ, Paschal J, et al. Incidence of hydroxychloroquine retinopathy in 1,207 patients in a large multicenter outpatient practice. Arthritis Rheum 1997:40:1482-6.

8 Shipley M, Silman A. Should patients on hydroxychloroquine have their eyes examined regularly? Br J Rheumatol 1997;36:514-5.

9 Hodis HN, Quismorio FP Jr, Wickham E, et al. The lipid, lipoprotein, and apolipoprotein effects of hydroxychloroquine in patients with systemic lupus erythematosus. J Rheumatol 1993;20:661-5.

10 loannou Y, Sultan S, Isenberg D. Causes of death in SLE: an analysis of 266 patients under long term review. Rheumatology 2000;39(abst 265).

11 Sen D, Keen RW. Osteoporosis in systemic lupus erythematosus: prevention and treatment. Lupus 2001;10:227-32.

12 Ramsey-Goldman R, Dunn JE, Huang CF, et al. Frequency of fractures in women with systemic lupus erythematosus: comparison with United States population data. Arthritis Rheum 1999:42:882-90.

13 Zonana-Nacach A, Barr SG, Magder LS, et al. Damage in systemic lupus erythematosus and its association with corticosteroids. Arthritis Rheum 2000;43:1801-8.

14 Saag KG, Emkey R, Schnitzer TJ, et al. Alendronate for the prevention and treatment of glucocorticoid-induced osteoporosis. Glucocorticoid-Induced Osteoporosis Intervention Study Group. N Engl J Med 1998:339:292-9.

15 Cohen S, Levy RM, Keller M, et al. Risedronate therapy prevents corticosteroid-induced bone loss: a twelve-month, multicenter, randomized, double-blind, placebo-controlled, parallel-group study. Arthritis Rheum 1999:42:2309-18.

16 Reid DM, Hughes RA, Laan RF, et al. Efficacy and safety of daily risedronate in the treatment of corticosteroid-induced osteoporosis in men and women: a randomized trial. European Corticosteroid-Induced Osteoporosis Treatment Study. J Bone Miner Res 2000;15:1006-13.

17 Boumpas DT, Austin HA 3rd, Vaughn EM, et al. Controlled trial of pulse methylprednisolone versus two regimens of pulse cyclophosphamide in severe lupus nephritis. Lancet 1992;340:741-5.

18 Illei GG, Austin HA, Crane M, et al. Combination therapy with pulse cyclophosphamide plus pulse methylprednisolone improves long-term renal outcome without adding toxicity in patients with lupus nephritis. Ann Intern Med 2001;135:248-57.

19 Bansal VK, Beto JA. Treatment of lupus nephritis: a meta-analysis of clinical trials. Am J Kidney Dis 1997;29:193-9.

20 Stone JH, Millward CL, Olson JL, et al. Frequency of recurrent lupus nephritis among ninety-seven renal transplant patients during the cyclosporine era. Arthritis Rheum 1998;41:678-86. 
21 Stone JH, Amend WJ, Criswell LA. Outcome of renal transplantation in ninety-seven cyclosporine-era patients with systemic lupus erythematosus and matched controls. Arthritis Rheum 1998;41:1438-45.

22 Weinshilboum RM, Sladek SL. Mercaptopurine pharmacogenetics: monogenic inheritance of erythrocyte thiopurine methyltransferase activity. Am J Hum Genet 1980;32:651-62.

23 Yates CR, Krynetski EY, Loennechen T, et al. Molecular diagnosis of thiopurine S-methyltransferase deficiency: genetic basis for azathioprine and mercaptopurine intolerance. Ann Intern Med 1997;126:608-14.

24 Naughton MA, Battaglia E, O'Brien S, et al. Identification of thiopurine methyltransferase (TPMT) polymorphisms cannot predict myelosuppression in systemic lupus erythematosus patients taking azathioprine. Rheumatology 1999;38:640-4.

25 Rahman P, Humphrey-Murto S, Gladman DD, et al. Efficacy and tolerability of methotrexate in antimalarial resistant lupus arthritis. J Rheumatol 1998;25:243-6.

26 Carneiro JR, Sato El. Double blind, randomized, placebo controlled clinical trial of methotrexate in systemic lupus erythematosus. J Rheumatol 1999;26: 1275-9.

27 Chan TM, Li FK, Tang CS, et al. Efficacy of mycophenolate mofetil in patients with diffuse proliferative lupus nephritis. Hong Kong-Guangzhou Nephrology Study Group. N Engl J Med 2000;343:1 156-62.

$28 \mathrm{McClure} \mathrm{CE}$, Isenberg DA. Does plasma exchange have any part to play in the management of SLE? In: Isenberg DA, Tucker LB, eds. Controversies in SLE. London: Martin Dunitz, 1997: 75-86.

29 Schroeder JO. Plasmaphoresis and subsequent pulse cyclophosphamide in severe systemic lupus erythematosus. Arthritis Rheum 1997 40(S):S325.
30 Walton AJ, Snaith ML, Locniskar M, et al. Dietary fish oil and the severity of symptoms in patients with systemic lupus erythematosus. Ann Rheum Dis 1991;50:463-6.

31 Hakim AJ, Machin SJ, Isenberg DA. Autoimmune thrombocytopenia in primary antiphospholipid syndrome and systemic lupus erythematosus: the response to splenectomy. Semin Arthritis Rheum 1998;28:20-5.

32 Boletis JN, loannidis JP, Boki KA, et al. Intravenous immunoglobulin compared with cyclophosphamide for proliferative lupus nephritis. Lancet 1999:354:569-70.

33 Isenberg DA, Snaith ML, Morrow WJ, et al. Cyclosporin A for the treatment of systemic lupus erythematosus. Int J Immunopharmacol 1981;3:163-9.

34 Caccavo D, Lagana B, Mitterhofer AP, et al. Long-term treatment of systemic lupus erythematosus with cyclosporin A. Arthritis Rheum 1997:40:27-35.

35 Mok CC, Wong RW. Pregnancy in systemic lupus erythematosus. Postgrad Med J 2001;77:157-65.

36 Rubin RL. Drug-induced lupus. In: Wallace DJ, Hahn BH, eds. Dubois lupus erythematosus. 5th Ed. Baltimore: Williams and Wilkins, 1997: $871-901$

37 Maini RN, Breedveld FC, Kalden JR, et al. Therapeutic efficacy of multiple intravenous infusions of anti-tumor necrosis factor alpha monoclonal antibody combined with low-dose weekly methotrexate in rheumatoid arthritis. Arthritis Rheum 1998:41:1552-63.

38 Traynor AE, Schroeder J, Rosa RM, et al. Treatment of severe systemic lupus erythematosus with high-dose chemotherapy and haemopotic stem-cell transplantation: a phase I study. Lancet 2000;356:701-7. 